\title{
Emprego do gel de plasma rico em plaquetas no tratamento de feridas em cães:
}

\section{revisão}

\author{
Use of platelet-rich plasma gel in the treatment of wounds in dogs: review \\ Uso de gel de plasma rico em plaquetas en el tratamento de heridas en perros: revisión
}

Recebido: 07/12/2021 | Revisado: 12/12/2021 | Aceito: 18/12/2021 | Publicado: 02/01/2022

\author{
Larissa Pereira Gonzaga \\ ORCID: https://orcid.org/0000-0003-3834-3511 \\ Universidade José do Rosário Vellano, Brasil \\ E-mail: larissamedvet2017@gmail.com \\ Vitória Fagioli Schutze \\ ORCID: https://orcid.org/0000-0002-1238-9311 \\ Universidade José do Rosário Vellano, Brasil \\ E-mail: mvvitoriafagiolis@gmail.com \\ Poliana Silva Beker dos Reis \\ ORCID: https://orcid.org/0000-0001-9913-9237 \\ Universidade José do Rosário Vellano, Brasil \\ E-mail: poliana.reis@prof.unifenas.br \\ Nelma de Mello Silva Oliveira \\ ORCID: https://orcid.org/0000-0002-1114-2018 \\ Universidade José do Rosário Vellano, Brasil \\ E-mail: nelma.oliveira@unifenas.br \\ José Antônio Dias Garcia \\ ORCID: https://orcid.org/0000-0002-4024-3045 \\ Universidade José do Rosário Vellano, Brasil \\ E-mail: jadiasgarcia@gmail.com
}

\begin{abstract}
Resumo
O plasma possui uma elevada concentração de plaquetas em um pequeno volume final. Denominado de Plasma Rico em Plaquetas (PRP), é considerado um hemocomponente que ao ser retirado do próprio paciente pode ser utilizado no tratamento de feridas tanto em humanos como em animais de diversas espécies, a fim de se obter uma cicatrização mais rápida. A cicatrização é um processo resultante da regeneração do tecido lesado, que ocorre através de uma cascata de eventos que se divide em três fases: a inflamação; a proliferação e por último o remodelamento tecidual. Este trabalho teve o objetivo revisar a literatura para elucidar melhor a eficácia do gel de plasma rico em plaquetas no tratamento de ferida de cães, através da resposta da cicatrização e da regeneração tecidual. Foi realizada uma busca por dissertações e artigos científicos nas linguagens portuguesa, espanhola e inglesa, publicados no intervalo de 1997 a 2019, a fim de reconhecer os efeitos do plasma rico em plaquetas, sua composição, modo de preparo e uso em feridas nos animais, especialmente nos cães. Pode se concluir que o tratamento de feridas em cães com o plasma possui bom efeito antimicrobiano, reduzindo risco de infecções, sendo de fácil aplicação, resultando em uma boa aderência na área da lesão e a um baixo custo em sua obtenção.
\end{abstract}

Palavras-chave: Cães; Cicatrização; Feridas; Plasma rico em plaquetas.

\begin{abstract}
Plasma has a high concentration of platelets in a small final volume. Called Platelet Rich Plasma (PRP), it is considered a blood component that, when removed from the patient, can be used in the treatment of wounds both in humans and animals of various species, in order to obtain faster healing. Healing is a process resulting from the regeneration of damaged tissue, which occurs through a cascade of events that is divided into three phases: inflammation; proliferation and finally tissue remodeling. This work aimed to review the literature to better elucidate the efficacy of platelet-rich plasma gel in the treatment of dog wounds, through the healing response and tissue regeneration. A search was carried out for dissertations and scientific articles in Portuguese, Spanish and English, published between 1997 and 2019, in order to recognize the effects of platelet-rich plasma, its composition, method of preparation and use in wounds in animals, especially in dogs. It can be concluded that the treatment of wounds in dogs with plasma has a good antimicrobial effect, reducing the risk of infections, being easy to apply, resulting in good adherence to the wound area and a low cost of obtaining it.
\end{abstract}

Keywords: Dogs; Healing; Wounds; Platelet-rich plasma. 


\begin{abstract}
Resumen
El plasma tiene una alta concentración de plaquetas en un pequeño volumen final. Denominado Plasma Rico en Plaquetas (PRP), se considera un componente sanguíneo que, extraído del paciente, puede utilizarse en el tratamiento de heridas tanto en humanos como en animales de diferentes especies, con el fin de obtener una cicatrización más rápida. La curación es un proceso resultante de la regeneración del tejido dañado, que ocurre a través de una cascada de eventos que se divide en tres fases: inflamación; proliferación y finalmente remodelación tisular. Este trabajo tuvo como objetivo revisar la literatura para dilucidar mejor la eficacia del gel de plasma rico en plaquetas en el tratamiento de heridas en perros, a través de la respuesta de cicatrización y la regeneración tisular. Se realizó una búsqueda de disertaciones y artículos científicos en portugués, español e inglés, publicados entre 1997 y 2019 , con el fin de reconocer los efectos del plasma rico en plaquetas, su composición, método de preparación y uso en heridas en animales, especialmente en perros. Se puede concluir que el tratamiento de heridas en perros con plasma tiene un buen efecto antimicrobiano, reduciendo el riesgo de infecciones, siendo de fácil aplicación, resultando en una buena adherencia a la zona de la herida y un bajo coste de obtención.
\end{abstract}

Palabras clave: Perros; Curación; Heridas; Plasma rico en plaquetas.

\title{
1. Introdução
}

O Plasma Rico em Plaquetas (PRP) é também encontrado na literatura como concentrado de plaquetas, plasma enriquecido em plaquetas, plasma rico em fatores de crescimento ou gel de plaquetas (Marx, 2004). É um derivado sanguíneo de fácil obtenção e com ótima relação custo/benefício, além de dispor de ação terapêutica cicatrizante que atuam na reparação e regeneração dos tecidos, sendo considerado uma opção útil no processo cicatricial de muitas enfermidades (Maia \& Souza, 2009).

$\mathrm{Na}$ medicina equina, sua utilização tem sido descrita em tratamento de feridas (Carter, Jolly, Worden Sr, Hendren, \& Kane, 2003), tendinites e desmites (Maia \& Souza, 2009; Sutter, 2008), osteoartrite (J U Carmona, Argüelles, Climent, \& Prades, 2007) e na cicatrização de fraturas (Jorge U Carmona \& López, 2011). Entretanto, embora seja bastante utilizado, ainda é raro um protocolo padrão estabelecido para a sua aquisição/utilização através da centrifugação do sangue total do animal (Nikolidakis \& Jansen, 2008).

Nas especialidades médicas humana, é utilizado em diversas áreas da cirurgia plástica e reconstrutiva, na otolaringologia e na odontologia, tornou um produto com grande potencial na integração de enxertos, sejam eles ósseos, cutâneos, cartilaginosos ou de gordura, bem como estimulante na cicatrização de feridas (Vendramin, Franco, \& Franco, 2009).

Estudos evidenciam a sua capacidade de atuar na síntese e liberação de fatores de crescimento (FC) e citocinas, que realizam a ação anti-inflamatória e antibacteriana promovendo a regeneração dos tecidos acometidos (Carneiro, Barbieri, \& Barbieri Neto, 2013). Embora o produto final resulte em um pequeno volume de plasma, este possui elevado número de plaquetas e fatores de crescimento justificando sua aplicabilidade na medicina terapêutica (Vendramin, Franco, Nogueira, Pereira, \& Franco, 2006).

A sua obtenção pode ser de forma manual, semiautomática ou por aférese (Prades, Abellanet, Carmona, Argüelles, \& Masri, 2006; Vendrusculo, Watanabe, Maia, Carvalho, \& Alves, (2012), onde após a coleta do sangue do paciente, é necessário que este passe por uma centrifugação resultando na separação das plaquetas (Vendrusculo et al., 2012). Para o processo é utilizada máquinas de plasmaferese e trombina bovina para a sua ativação, mas estudos relatam variados protocolos com substituição da trombina bovina por trombina autóloga e máquinas automatizadas com kits que facilitam a obtenção do PRP, mas ainda bem onerosos (Vendramin et al., 2006). Ainda segundo o autor, é possível realizar de forma mais econômica e em locais com menos recursos, mas é necessário que o profissional tenha treinamento específico, além disso, precisa-se de pelo menos uma centrífuga e materiais como seringas, agulhas e tubos de coleta de sangue. 
Considerando seu uso em feridas descritos na literatura, como vantajosos por seu efeito antimicrobiano e redução do risco de infecções (Bielecki et al., 2007), fácil aplicação, ótima aderência na região da lesão e baixo custo(Yamada et al., 2012; Pinto \& Pizani, 2015), sua importância no processo de cicatrização e regeneração tecidual no aspecto da clínica veterinária, buscamos realizar uma revisão bibliográfica sobre o tema a fim de discorrer sobre a utilização do gel de PRP para o tratamento de feridas em cães, analisando o processo de cicatrização e regeneração tecidual a fim de comprovar sua eficácia através da comparação de estudos já realizados antes.

\section{Metodologia}

Foi realizada uma revisão de literatura narrativa (Rother, 2007; Faculdade de Ciências Agronomicas, 2015), elaborada por meio de levantamento dos artigos científicos publicados entre o período de 1997 a 2019 a fim de discorrer sobre os efeitos do plasma rico em plaquetas analisando sua composição, modo de preparo e seu uso em feridas em animais, especialmente em cães. Foram utilizados os buscadores Elsevier, Google Academic, Periódicos Capes, Science Direct e Medline, usando os seguintes filtros: cicatrização de feridas; plasma rico em plaquetas e tratamentos com plasma rico em plaquetas. Para a seleção foram utilizados como critérios de inclusão estudos publicados tanto em Língua Inglesa, espanhola e Portuguesa, e a relevância para a temática. Foram excluídos trabalhos sem embasamento científico ou aqueles cujos dados não tenham sido disponibilizados por completo.

\section{Resultados e Discussão}

A reparação de tecidos lesionados é uma resultante que ocorre de etapas, sendo elas, a fase exsudadtiva, fase de granulação e fase de remodelamento ou reparativa, com objetivo para restaurar a função e estruturas normais. A fase exsudativa surge no momento da lesão, pois juntamente ao sangramento chegam a ativação das proteases do plasma dos sistemas da coagulação (as plaquetas, hemácias e fibrina), que irão vedar as bordas da ferida fazendo com que tenha início os processos de adesão e agregação celular. (Tazima, De Andrade Vicente, \& Moriya, 2008). Um coágulo será formado servindo como um tampão hemostático e não havendo mais perda sanguínea e impermeabilizando para evitar contaminação (Isaac, Ladeira, Rêgo, Aldunate, \& Ferreira, 2010). Acontece também a liberação local mediadores inflamatórios (histamina, serotonina e bradicinina), que irão promover o surgimento de Fenômenos Vasculares que irão desencadear vasodilatação e aumento de fluxo sanguíneo local, além de sinais inflamatórios como o calor e rubor e aumento da permeabilidade capilar levando o extravasamento de líquidos para o espaço extracelular, gerando um edema (Balbino, Pereira, \& Curi, 2005; Jessop et al., 2010). A resposta inflamatória decorre durante três dias, onde através dos mediadores bioquímicos verifica-se a migração de células para a ferida, aumentando a permeabilidade vascular, ocorrendo a exsudação plasmática e passagem de elementos celulares para a área da ferida. A prostaglandina é um dos mediadores mais importantes no processo de cicatrização, pois favorece a exsudação vascular, estimulando a mitose celular e a quimiotaxia de leucócitos (Tazima et al., 2008).

A fase granulação é composta de três eventos: neoangiogênese, fibroplasia e epitelização. Fase essa caracterizada pela formação de tecido de granulação, formado por um leito capilar, fibroblastos, macrófagos, um arranjo frouxo de colágeno, fibronectina e ácido hialurônico (Isaac et al., 2010). É iniciada por volta do $3^{\circ}$ dia após a lesão, permanecendo por 2 a 3 semanas e é o marco inicial da formação da cicatriz. A neoangiogênese é o processo de formação de novos vasos sanguíneos, útil para manter o ambiente de cicatrização da ferida. Já a fibroplasia acontece após o trauma, onde células mesenquimais normalmente quiescentes e esparsas no tecido normal, são transformadas em fibroblastos e atraídas para o local inflamatório, que irão se dividir e produzem os componentes da matriz extracelular (George Broughton, Janis, \& Attinger, 2006). No 
entanto, o fibroblasto só aparece no sítio da lesão a partir do $3^{\circ}$ dia, onde os leucócitos polimorfonucleares já fizeram sua função, higienizando a área traumatizada (Tazima et al., 2008). A principal função dos fibroblastos é sintetizar colágeno, ainda na fase celular da inflamação. Por fim, na epitelização que inicia nas primeiras 24 a 36 horas após a lesão, fatores de crescimento epidérmicos estimulam a proliferação de células do epitélio. Na pele os ceratinócitos são capazes de sintetizar diversas citocinas que estimulam a cicatrização das feridas cutâneas (Laureano \& Rodrigues, 2011).

Para finalização do processo, acontece a fase de maturação, onde a ferida sofre um processo de contração por meio de um movimento centrípeto de toda a espessura da pele circundante, reduzindo então a sua quantidade e tamanho (Tazima et al., 2008). Processo muito importante aliado da cicatrização das feridas, principalmente nas abertas. Entretanto, se ocorrer de forma exagerada e desordenada causa defeitos cicatriciais importantes devido a diferenciação dos fibroblastos em miofibroblastos, estimulados por fatores decrescimento (George Broughton et al., 2006; Singer \& Clark, 1999). A maturação da ferida tem início durante a $3^{a}$ semana e caracteriza-se por um aumento da resistência, sem aumento na quantidade de colágeno. Há então um equilíbrio de produção e destruição das fibras de colágeno neste período, por ação da colagenase (Tazima et al., 2008). Já o desequilíbrio desta relação favorece cicatrizes hipertróficas e queloides (Gurtner, Werner, Barrandon, \& Longaker, 2008). Essa última fase dura toda a vida da ferida, embora o aumento da força tênsil se estabilize, após um ano, em 70 a $80 \%$ da pele intacta. A inclinação da curva de maturação é mais aguda durante as primeiras seis a oito semanas (Medeiros \& Dantas-Filho, 2016).

As feridas podem ser classificadas de três formas diferentes de acordo com o agente causal, o grau de contaminação e o comprometimento tecidual. Quanto ao agente causal podem ser divididas em incisas ou cirúrgicas (produzidas por um instrumento cortante); contusas (são produzidas por objeto rombo e caracterizadas por traumatismo das partes moles, hemorragia e edema); lacerantes (são ferimentos com margens irregulares e com mais de um ângulo); perfurantes (são caracterizadas por pequenas aberturas na pele). Para a classificação do grau de contaminação, as feridas limpas são as que não apresentam sinais de infecção e não sendo atingidos o trato respiratório, digestivo, genital e urinário, ou seja, baixa probabilidade de infecção em torno de 1 a $5 \%$. As feridas classificadas como infectadas são aquelas que apresentam sinais nítidos de infecção; as limpa-contaminadas são os ferimentos que possuem contaminação grosseira, havendo contato com o trato respiratório, digestivo, urinário e genital, porém em situações controladas, sendo o risco de infecção é cerca de $10 \%$; e as feridas tidas como contaminadas geralmente são feridas acidentais, com mais de seis horas de trauma ou que tiveram contato com terra e fezes, por exemplo, onde os riscos de infecção podem atingir 20 a $30 \%$. E por fim, o comprometimento tecidual é dividido em estágio I (comprometimento da epiderme apenas, sem perda tecidual); estágio II (ocorre perda tecidual e comprometimento da epiderme, derme ou ambas); estágio III (há comprometimento total da pele e necrose de tecido subcutâneo, mas não atinge a fáscia muscular); o estágio IV, há extensa destruição de tecido, chegando a ocorrer lesão óssea ou muscular ou necrosetissular (Tazima et al., 2008; Santos et al., 2009).

Atualmente, sabe-se que as plaquetas podem secretar substâncias reparadoras de tecidos como proteínas, fator de crescimento e citocinas que vão ativar e convocar as células do sistema imune dos animais e humanos, como os neutrófilos, macrófagos e fibroblastos. Estas células irão se ligar à matriz extracelular ativando os macrófagos, interleucinas como a $I L-1 \alpha$, $I L-1 \beta$ e fator de necrose tumoral $(T N F-\alpha)$, ativando a síntese de colágeno e secreção de fator de crescimento de fibroblasto-2, fator de crescimento semelhante à insulina-1 e fator de crescimento transformador beta-1 e 2. Para acontecer a angiogênese serão secretados o fator crescimento do endotélio vascular e fator de crescimento derivado de plaquetas (Dreifke, Jayasuriya, \& Jayasuriya, 2015). 
Frente as funções que as plaquetas realizam, o plasma rico em plaquetas é um produto biológico que vem sendo utilizado na medicina, seja ela humana ou veterinária sendo bastante eficiente em promover desde regeneração e revascularização do tecido até a reepitelização e fechamento de feridas em diversos tipos de tecidos. Sendo empregado na ortopedia, na cirurgia e implantodontia buco-maxilo-facial, oftalmologia e dermatologia (Etulain, 2018).

O PRP é feito através da coleta de sangue do paciente e centrifugação do mesmo, com o intuito de separar as plaquetas (Marx, 2004). Em estudos com sangue de cães foi descrito como executável e de fácil manuseio, além de concentrar as plaquetas de forma adequada. O gel formado apresentou consistência adequada para utilização clínica e, a avaliação preliminar de uso demonstra resultados extremamente promissores (Barbosa et al., 2008). Quanto a concentração de plaquetas obtidas no trabalho de Vanat, da Silva Medeiros, Balarin, Pereira, \& De Biasi, (2012), foi possível mesmo com um volume pequeno de sangue inicial, possibilitando a utilização do PRP autólogo que oferece vantagem ao PRP heterólogo, como também citado por (Kasten et al., 2008).

Estudos com ferida após excisão de massa neoplásica tratada com plasma rico em plaquetas, demonstrou diminuição a resposta inflamatória, os bordos mais uniformes, sem secreção purulenta, sem tecido de granulação exuberante e ausência de complicações. No entanto, o mesmo estudo relatou a dificuldade de análise de resultados devido à ausência de técnica padrão no preparo do tratamento (Vidal \& de Souza Zat, 2019). Nos trabalhos de Tambella et al., (2018) e Martins et al., (2012), também foi relatado a diminuição da resposta inflamatória, tecido de granulação excessivo ausente, com cicatrização mais limpa e bordo da ferida mais uniforme, corroborando com (Vidal \& de Souza Zat, 2019), que sugere a utilização do produto em animais com a clínica parecida e com amostragem maior.

Em contrapartida aos estudos anteriores, no estudo de Bonfá et al., (2017), concluiu que a taxa de sobrevivência dos enxertos no pescoço de equinos foi boa, no intervalo de 63 a 75\%, porém, não houve diferenças significativas entre a região tratada com gel de plasma rico em plaquetas e região de controle, através de análises clínicas e histológicas. Além disso, o índice de falhas na enxertia foi de $37,5 \%$ no lado controle e de $25 \%$ no lado tratado com o gel. Este mesmo fato foi observado por outros autores como Monteiro, Lepage, \& Theoret., (2009) e DeRossi et al., (2009), todos os dois realizaram procedimento em feridas de equinos. Além disso, um outro trabalho feito em enxertos de cães também descreveu o mesmo resultado, sendo que o tecido que foi utilizado a cola de fibrina teve melhor resultado que o gel de plasma rico em plaquetas (Hermeto, Rossi, Pádua, Pontes, \& Santana, 2012).

As feridas tratadas com o PRP em membros podem fechar em até 72 dias no máximo, o que mostra sem resultado controverso, pois as feridas de membros sem esse tratamento demoram cerca de 62 dias (Monteiro, Lepage, \& Theoret, 2009). Segundo Ferreira \& Costa, (2007), as feridas após cirurgia com esse tratamento demoram em torno de 37 dias para fechar, enquanto nos animais não tratados demoram até 38 dias e meio.

É importante mencionar que a cicatrização das lesões depende muito do seu diâmetro e da região afetada, logo que as feridas de membros têm mais dificuldade na cicatrização, enquanto as localizadas em no tronco são mais(Wilmink \& van Weeren, 2005). E que independentemente do método escolhido, o anticoagulante será o citrato de sódio, pois não irá alterar os receptores de membrana das plaquetas. O citrato de sódio obtém os íons de cálcio do sangue neutralizando-os para formar o quelato e assim bloquear o aparecimento de coágulo (Anitua \& Ortiz, 2000).

\section{Conclusão}

De acordo com a revisão de literatura, o gel de PRP possui um bom fator cicatricial, podendo ser considerado uma boa escolha no tratamento de feridas na espécie canina. Alguns estudos ainda são controversos, entretanto seu uso é descrito como benéfico e trazendo avanços promissores quanto ao tempo de regeneração tecidual na medicina humana, o que sugere o 
uso dessa técnica deve ainda haver maior divulgação e estudos científicos na medicina veterinária, objetivando sempre o aprimoramento da técnica. Muitas vezes é necessário complementar o tratamento, seja por via sistêmica ou por via tópica, a fim de obter um melhor resultado na redução da inflamação local ou sistêmica, cicatrização e remodelação tecidual, adiantando e facilitando a melhora total dos animais.

\section{Referências}

Anitua, A., \& Ortiz, A. (2000). Un nuevo enfoque en la regeneración ósea. Plasma rico en factores de crecimiento. Victoria, Spain, Editorial Puesta Al Dıa.

Balbino, C. A., Pereira, L. M., \& Curi, R. (2005). Mechanisms involved in wound healing: A revision. Revista Brasileira de Ciências Farmacêuticas, 41(1), 27-51. https://doi.org/10.1590/S1516-93322005000100004

Barbosa, A. L. T., Del Carlo, R. J., Gomes, H. C., Oliveira, A. C. de, Monteiro, B. S., \& Del Carlo, B. N. (2008). Plasma rico em plaquetas para reparação de falhas ósseas em cães. Ciência Rural, 38, 1335-1340.

Bielecki, T. M., Gazdzik, T. S., Arendt, J., Szczepanski, T., Krol, W., \& Wielkoszynski, T. (2007). Antibacterial effect of autologous platelet gel enriched with growth factors and other active substances: an in vitro study. The Journal of Bone and Joint Surgery. British Volume, 89(3), 417-420.

Bonfá, A. F., Nomura, R. H. C., Prado, A. M. B. do, Silveira, A. B. da, Dornbusch, L. P. T. C., \& Dornbusch, P. T. (2017). Efeito do gel de plasma rico em plaquetas na cicatrização de enxertos cutâneos em equinos. Ciência Animal Brasileira, 18.

Carmona, J. U, Argüelles, D., Climent, F., \& Prades, M. (2007). Autologous platelet concentrates as a treatment of horses with osteoarthritis: a preliminary pilot clinical study. Journal of Equine Veterinary Science, 27(4), 167-170.

Carmona, Jorge U, \& López, C. (2011). Autologous platelet concentrates as a treatment for shoulder injury in a horse. Journal of Equine Veterinary Science, $31(9), 506-510$.

Carneiro, M. de O., Barbieri, C. H., \& Barbieri Neto, J. (2013). O gel de plasma rico em plaquetas propicia a regeneração da cartilagem articular do joelho de ovelhas. Acta Ortopédica Brasileira, 21, 80-86.

Carter, C. A., Jolly, D. G., Worden Sr, C. E., Hendren, D. G., \& Kane, C. J. M. (2003). Platelet-rich plasma gel promotes differentiation and regeneration during equine wound healing. Experimental and Molecular Pathology, 74(3), 244-255.

Rossi, R., Coelho, A. C. A. de O., Mello, G. S. de, Frazílio, F. O., Leal, C. R. B., Facco, G. G., \& Brum, K. B. (2009). Effects of platelet-rich plasma gel on skin healing in surgical wound in horses. Acta Cirúrgica Brasileira, 24, 276-281.

Dreifke, M. B., Jayasuriya, A. A., \& Jayasuriya, A. C. (2015). Current wound healing procedures and potential care. Materials Science and Engineering: C, $48,651-662$.

Etulain, J. (2018). Platelets in wound healing and regenerative medicine. Platelets, 29(6), 556-568.

Faculdade de Ciências Agronomicas. (2015). Tipos de revisão de literatura. Faculdade de Ciências Agronomicas UNESP Campus de Botucatu, 9. Retrieved from http://www.fca.unesp.br/Home/Biblioteca/tipos-de-evisao-de-literatura.pdf

Ferreira, J. C., \& Costa, J. C. M. (2007). Efeitos da monofenilbutazona em eqüinos: cicatrização por segunda intenção e lesão muscular. Revista Ceres, (311313), 263.

George Broughton, I. I., Janis, J. E., \& Attinger, C. E. (2006). The basic science of wound healing. Plastic and Reconstructive Surgery, 117(7S), 12S-34S.

Gurtner, G. C., Werner, S., Barrandon, Y., \& Longaker, M. T. (2008). Wound repair and regeneration. Nature, 453(7193), 314-321.

Hermeto, L. C., Rossi, R. de, Pádua, S. B. de, Pontes, E. R. J., \& Santana, A. E. (2012). Estudo comparativo entre a cola de fibrina eo plasma rico em plaquetas em enxertos cutâneos em cães. Acta Cirúrgica Brasileira, 27(11), 789-794.

Isaac, C., Ladeira, P. R. S. de, Rêgo, F. M. P. do, Aldunate, J. C. B., \& Ferreira, M. C. (2010). Processo de cura das feridas: cicatrização fisiológica. Revista de Medicina, 89(3/4), 125. https://doi.org/10.11606/issn.1679-9836.v89i3/4p125-131

Jessop, D. S., Fassold, A., Wolff, C., Hofbauer, R., Chover-Gonzalez, A., Richards, L. J., \& Straub, R. H. (2010). Endomorphins in rheumatoid arthritis, osteoarthritis, and experimental arthritis: Annals of the New York Academy of Sciences. Annals of the New York Academy of Sciences, 1193, 117-122. https://doi.org/10.1111/j.1749-6632.2009.05294.x

Kasten, P., Vogel, J., Geiger, F., Niemeyer, P., Luginbühl, R., \& Szalay, K. (2008). The effect of platelet-rich plasma on healing in critical-size long-bone defects. Biomaterials, 29(29), 3983-3992.

Laureano, A., \& Rodrigues, A. M. (2011). Cicatrização de feridas. Journal of the Portuguese Society of Dermatology and Venereology, $69(3), 355$.

Maia, L., \& Souza, M. V. de. (2009). Componentes ricos em plaquetas na reparação de afecções tendo-ligamentosas e osteo-articulares em animais. Ciência Rural, 39, 1267-1274.

Martins, R. K., Matheus, J. P., Klug, F. S. F., Furtado, T. C., Malschitzky, E., de Oliveira, S. J., ... Allgayer, M. D. A. C. (2012). Tratamento clínico de lesões 
Research, Society and Development, v. 11, n. 1, e9111124479, 2022

(CC BY 4.0) | ISSN 2525-3409 | DOI: http://dx.doi.org/10.33448/rsd-v11i1.24479

cutâneas em equinos com plasma rico em plaquetas (prp)-resultados preliminares. In xviii salão de iniciação científica e tecnológica.

Marx, R. E. (2004). Platelet-rich plasma: evidence to support its use. Journal of Oral and Maxillofacial Surgery, 62(4), 489-496.

Medeiros, A. C., \& Dantas-Filho, A. M. (2016). Cicatrização das feridas cirúrgicas. Journal of Surgical and Clinical Research, 7(2), 87-102.

Monteiro, S. O., Lepage, O. M., \& Theoret, C. L. (2009). Effects of platelet-rich plasma on the repair of wounds on the distal aspect of the forelimb in horses. American Journal of Veterinary Research, 70(2), 277-282.

Nikolidakis, D., \& Jansen, J. A. (2008). The biology of platelet-rich plasma and its application in oral surgery: literature review. Tissue Engineering Part B: Reviews, 14(3), 249-258.

Pinto, J. M. N., \& Pizani, N. S. (2015). Aplicabilidade em dermatologia do plasma rico em plaquetas. Surgical \& Cosmetic Dermatology, 7(1), 61-64.

Prades, M., Abellanet, I., Carmona, J. U., Argüelles, D., \& Masri, M. (2006). Platelet rich plasma: a realistic alternative in tissue repair. In 15th Annual Meeting European College of Veterinary Surgeons (pp. 211-216).

Rother, E. T. (2007). Revisão sistemática X revisão narrativa. Acta Paulista de Enfermagem, 20(2), v-vi. https://doi.org/10.1590/s0103-21002007000200001

Santos, J. B. dos, Porto, S. G., Suzuki, L. M., Da, L., Sostizzo, R. Z., Antoniazzi, J. L., \& Echer, I. C. (2009). Avaliação e tratamento de feridas: Orientação aos profissionais de enfermagem. Hospital de Clínicas. Porto Alegre - RS, 44.

Singer, A. J., \& Clark, R. A. F. (1999). Cutaneous wound healing. New England Journal of Medicine, 341(10), 738-746.

SUTTER, W. W. (2008). Intralesional injection of platelet-rich plasma for mid-body suspensory ligament desmitis in Standardbred race horses. Journal American Veterinary Medical Association, 232(10), 1515-1520.

Tambella, A. M., Attili, A. R., Dupre, G., Cantalamessa, A., Martin, S., Cuteri, V., ... Del Fabbro, M. (2018). Platelet-rich plasma to treat experimentallyinduced skin wounds in animals: A systematic review and meta-analysis. PLoS One, 13(1), e0191093.

Tazima, M. D. F. G. S., De Andrade Vicente, Y. A. M. V., \& Moriya, T. (2008). Biologia da ferida e cicatrização. Medicina, 41(3), 255-260. https://doi.org/10.11606/issn.2176-7262.v41i3p259-264

Vanat, N., da Silva Medeiros, T. N., Balarin, M. R. S., Pereira, P. M., \& De Biasi, F. (2012). Modificação de técnica de preparo do plasma rico em plaquetas em cães. Semina: Ciências Agrárias, 33(1), 313-321.

Vendramin, F. S., Franco, D., \& Franco, T. R. (2009). Método de obtenção do gel de plasma rico em plaquetas autólogo. Rev. Bras. Cir. Plást, $212-218$.

Vendramin, F. S., Franco, D., Nogueira, C. M., Pereira, M. S., \& Franco, T. R. (2006). Plasma rico em plaquetas e fatores de crescimento: técnica de preparo e utilização em cirurgia plástica. Revista Do Colégio Brasileiro de Cirurgiões, 33, 24-28.

Vendrusculo, C. do P., Watanabe, M. J., Maia, L., Carvalho, A. M. de, \& Alves, A. L. G. (2012). Plasma rico em plaquetas: Uma nova perspectiva terapêutica para medicina equina. Veterinária e Zootecnia, 33-43.

Vidal, J. M., \& de Souza Zat, L. H. (2019). Utilização de plasma rico em plaquetas no tratamento de ferida cutânea em cão: relato de caso. Arquivos de Ciências Veterinárias e Zoologia Da UNIPAR, 22(2).

Wilmink, J. M., \& van Weeren, P. R. (2005). Second-intention repair in the horse and pony and management of exuberant granulation tissue. Veterinary Clinics: Equine Practice, 21(1), 15-32.

Yamada, A. L. M., Carvalho, A. M. de, Oliveira, P. G. G., Felisbino, S. L., Queiroz, D. L., Watanabe, M. J., ... Alves, A. L. G. (2012). Plasma rico em plaquetas no tratamento de lesões condrais articulares induzidas experimentalmente em equinos: avaliação clínica, macroscópica, histológica e histoquímica. Arquivo Brasileiro de Medicina Veterinária e Zootecnia, 64, 323-332. 